\title{
Analysis of deformed microstrip resonator using the finite element method
}

\author{
A S CHAUDHARI and P B PATIL* \\ Department of Physics, Dr B A M University, Aurangabad 431 004, India \\ MS received 20 July 1996; revised 21 May 1997
}

\begin{abstract}
Microstrip resonator with shape deformation has been analysed using the finite element method (FEM). Keeping the front surface of the microstrip resonator fixed, the back surface is deformed. Five cases of the deformation of the back surface are considered: (i) the left vertical side of the back surface is shifted inward; (ii) both vertical sides of the back surface are shifted inward; (iii) the right vertical side of the back surface is shifted outward; (iv) both vertical sides of the back surface are shifted outward; (v) left vertical side of back surface is shifted inward and the right vertical side of it is shifted outward. Variation in cutoff frequency for the $\mathrm{TM}_{011}, \mathrm{TM}_{110}, \mathrm{TM}_{111}, \mathrm{TM}_{012}, \mathrm{TM}_{112}$ and $\mathrm{TM}_{210}$ is observed.
\end{abstract}

Keywords. Microstrip resonator; finite element method.

\section{Introduction}

Finite element method (FEM) is a powerful tool for the approximate solutions of differential equations governing diverse physical problems. It can tackle problems of different branches in physics and it can be used for any complicated geometry or domain of regular or irregular shape. The method can deal with the problems described by coupled equation. The method is useful for inhomogeneous and anisotropic media also.

The resonant frequencies of open microstrip ring resonators are determined by Wolff \& Menzel (1975) and Pintzos \& Pregla (1978). The microstrip resonator with equilateral triangular patch is studied by Wolf \& Knoppic (1974), Helszajn et al (1979), Lyon \& Helszajn (1982). The triangular and rectangular patch microstrip resonator has been analysed by Kalamse \& Patil (1994) using the finite element method.

In this paper we have analysed the microstrip resonator with shape deformation for five cases:

(i) the left vertical side of the back surface is shifted inward;

(ii) both vertical sides of the back surface are shifted inward;

*For correspondence 
(iii) the right vertical side of the back surface is shifted outward;

(iv) both vertical sides of the back surface are shifted outward;

(v) left vertical side of back surface is shifted inward and the right vertical side of it is shifted outward.

\section{Statement of the problem}

Consider the rectangular microstrip resonator bounded by six faces B1, B2, B3, B4, B5, $\mathrm{B} 6$, as shown in figure $1 \mathrm{a}$.

The two side surfaces $\mathrm{B}_{1}, \mathrm{~B}_{2}$ and front and back surfaces B3, B4 are magnetic walls. The top and the bottom surfaces are electric walls.

The electric field $\mathbf{E}$ within the resonator will satisfy Maxwell's equations,

$$
\text { Curl Curl } \mathbf{E}-K^{2} \mathbf{E}=0 \text {, }
$$

where $K^{2}=\omega^{2} \mu_{0} \varepsilon_{0}$

$$
\operatorname{grad} \operatorname{div} \mathbf{E}-\nabla^{2} \mathbf{E}-K^{2} \mathbf{E}=0 .
$$

Since the medium is charge free, $\operatorname{div} \mathbf{E}=0$.

$$
\nabla^{2} \mathbf{E}+K^{2} \mathbf{E}=0 .
$$

The electric field within the substrate has only the $Z$ component and the magnetic field has the $X$ and $Y$ components. The tangential component of the magnetic field at the edge is negligible,

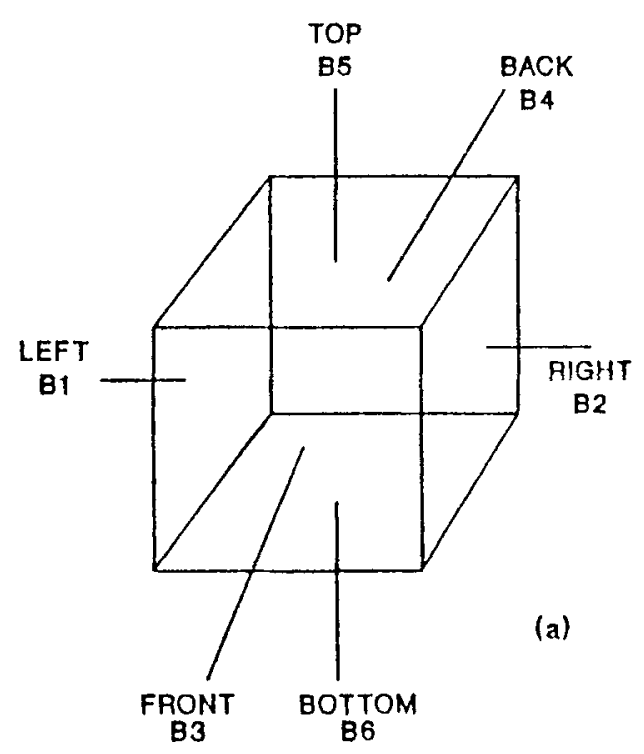

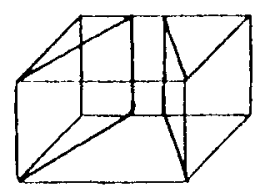

(c)

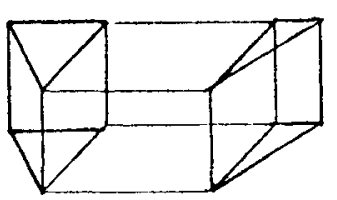

(e)

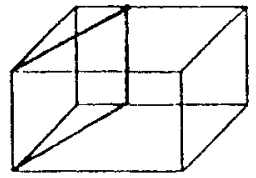

(b)

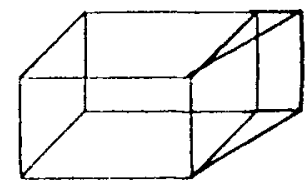

(d)

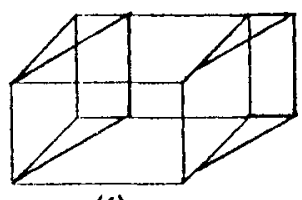

(f)

Figure 1. (a) Microstrip resonator without deformation. (b) Left vertical side of the back surface is shifted inward. (c) Both vertical sides of the back surface are shifted inward. (d) Right vertical side of the back surface is shifted outward. (e) Both vertical sides of the back surface are shifted outward. (f) Left vertical side of the back surface is shifted inward and right vertical side of the back surface is shifted outward. 


$$
\operatorname{div} \operatorname{grad} E_{z}+K^{2} E_{z}=0 .
$$

The fields within the resonator corresponding to TM modes will be generated by the equation,

$$
\nabla^{2} E_{z}+K^{2} E_{z}=0
$$

subjected to the boundary condition

$$
\begin{aligned}
& \left.\frac{\partial E_{z}}{\partial n}\right|_{\mathrm{B}_{1}, \mathrm{~B}_{2}, \mathrm{~B}_{3}, \mathrm{~B}_{4}}=0, \\
& \left.E_{z}\right|_{\mathrm{B}_{5}, \mathrm{~B}_{6}}=0,
\end{aligned}
$$

where $E_{z}$ is the $z$ component of $E$, and $\partial / \partial n$ represents normal derivative.

\section{Variational formulation}

To get the expression for the functional $\Pi$ in variational formulation, multiply (4) by some weight function $V$ and integrate it over the domain of the resonator,

$$
\Pi=\iint_{\Omega} \int V \operatorname{div} \operatorname{grad} E_{z} \mathrm{~d} \Omega+K^{2} \iint_{\Omega} \int V E_{z} \mathrm{~d} \Omega .
$$

Using the vector identity $S \operatorname{div} \mathbf{A}=\operatorname{div}(S \mathbf{A})-(\operatorname{grad} S) \cdot \mathbf{A}$ for the first term, $(8)$ becomes

$$
\begin{aligned}
\Pi= & \iint_{\Omega} \int \operatorname{div}\left(V \operatorname{grad} E_{z}\right) \mathrm{d} \Omega-\iint_{\Omega} \int(\operatorname{grad} V)\left(\operatorname{grad} E_{z}\right) \mathrm{d} \Omega \\
& +K^{2} \iint_{\Omega} \int V E_{z} \mathrm{~d} \Omega .
\end{aligned}
$$

Applying Gauss Divergence theorem to the first term in (9), we get

$$
\begin{aligned}
\Pi= & -\iint_{\Omega} \int(\operatorname{grad} V) \cdot\left(\operatorname{grad} E_{z}\right) \mathrm{d} \Omega+K^{2} \iint_{\Omega} \int V E_{z} \mathrm{~d} \Omega \\
& +\int_{S} \int V \operatorname{grad} E_{z} \cdot \bar{n} \mathrm{~d} s .
\end{aligned}
$$

Using (6), the last term in (10) will vanish.

Table 1. Variation of cutoff frequency for different modes for the inner deformation of one vertical side.

\begin{tabular}{lllllll}
\hline Shift & \multicolumn{7}{c}{ Frequency $(\mathrm{GHz})$} \\
\cline { 2 - 7 }$L(\mathrm{~cm})$ & $\mathrm{TM}_{011}$ & $\mathrm{TM}_{110}$ & $\mathrm{TM}_{111}$ & $\mathrm{TM}_{012}$ & $\mathrm{TM}_{112}$ & $\mathrm{TM}_{210}$ \\
\hline 0.05 & 13.607 & 14.016 & 15.005 & 16.470 & 17.641 & 17.906 \\
0.10 & 13.606 & 14.046 & 15.035 & 16.470 & 17.663 & 17.992 \\
0.15 & 13.605 & 14.077 & 15.066 & 16.470 & 17.685 & 18.073 \\
0.20 & 13.604 & 14.107 & 15.100 & 16.470 & 17.707 & 18.148 \\
0.25 & 13.603 & 14.139 & 15.136 & 16.470 & 17.728 & 18.219 \\
0.30 & 13.601 & 14.170 & 15.175 & 16.470 & 17.749 & 18.287 \\
0.35 & 13.599 & 14.202 & 15.217 & 16.471 & 17.770 & 18.351 \\
0.40 & 13.597 & 14.233 & 15.261 & 16.473 & 17.792 & 18.413 \\
\hline
\end{tabular}


Table 2. Variation of cutoff frequency for different modes for the inner deformation of both vertical sides.

\begin{tabular}{lllllll}
\hline Shift & \multicolumn{7}{c}{ Frequency $(\mathrm{GHz})$} \\
\cline { 2 - 7 }$L(\mathrm{~cm})$ & $\mathrm{TM}_{011}$ & $\mathrm{TM}_{110}$ & $\mathrm{TM}_{111}$ & $\mathrm{TM}_{012}$ & $\mathrm{TM}_{112}$ & $\mathrm{TM}_{210}$ \\
\hline 0.05 & 13.607 & 14.045 & 15.035 & 16.469 & 17.667 & 17.990 \\
0.10 & 13.607 & 14.103 & 15.104 & 16.468 & 17.719 & 18.130 \\
0.15 & 13.607 & 14.161 & 15.184 & 16.466 & 17.778 & 18.269 \\
0.20 & 13.608 & 14.218 & 15.277 & 16.464 & 17.842 & 18.379 \\
0.25 & 13.608 & 14.272 & 15.386 & 16.461 & 17.915 & 18.477 \\
0.30 & 13.609 & 14.324 & 15.513 & 16.458 & 17.998 & 18.567 \\
0.35 & 13.611 & 14.372 & 15.659 & 16.455 & 18.093 & 18.650 \\
0.40 & 13.612 & 14.417 & 15.825 & 16.451 & 18.203 & 18.728 \\
\hline
\end{tabular}

By substituting $V=E_{z}^{*}$ and changing the sign, (10) becomes

$$
\Pi=\frac{1}{2} \iint_{\Omega} \int\left(\operatorname{grad} E_{z}\right) \cdot\left(\operatorname{grad} E_{z}^{*}\right) \mathrm{d} \Omega-K^{2} \frac{1}{2} \iint_{\Omega} \int E_{z} E_{z}^{*} \mathrm{~d} \Omega .
$$

$\frac{1}{2}$ is introduced since $\Pi$ is bilinear functional.

The first variation $\delta \Pi$ is given by,

$$
\delta \Pi=\delta \frac{1}{2}\left[\iint_{\Omega} \int\left(\nabla E_{z}\right) \cdot\left(\nabla E_{z}^{*}\right) \mathrm{d} \Omega-K^{2} \iint_{\Omega} \int E_{z} E_{z}^{*} \mathrm{~d} \Omega\right] .
$$

For $\Pi$ to be stationary, $\delta \Pi$ should be minimum.

\section{Discretization}

According to the finite element method (Reddy 1986; Akin 1988), the volume of the resonator is divided into hexahedral elements with 20 nodes. The mapping functions assumed for these elements are quadratic in nature. The functional over an element is given by

$$
\Pi^{e}=\sum_{\text {ele }} \frac{1}{2}\left\{E_{z}^{e}\right\}^{T}\left[S^{e}\right]\left\{E_{z}^{e}\right\}-\frac{1}{2} K^{2} \sum_{\text {ele }}\left\{E_{z}^{e}\right\}^{T}\left[T^{e}\right]\left\{E_{z}^{e}\right\}
$$

Table 3. Variation of cutoff frequency for different modes for the outer deformation of one vertical side.

\begin{tabular}{lllllll}
\hline Shift & \multicolumn{7}{c}{ Frequency $(\mathrm{GHz})$} \\
\cline { 2 - 5 }$L$ (cm) & $\mathrm{TM}_{011}$ & $\mathrm{TM}_{110}$ & $\mathrm{TM}_{111}$ & $\mathrm{TM}_{012}$ & $\mathrm{TM}_{112}$ & $\mathrm{TM}_{210}$ \\
\hline 0.05 & 13.607 & 13.957 & 14.950 & 16.470 & 17.595 & 17.719 \\
0.10 & 13.606 & 13.930 & 14.925 & 16.469 & 17.570 & 17.622 \\
0.15 & 13.604 & 13.903 & 14.902 & 16.469 & 17.551 & 17.515 \\
0.20 & 13.602 & 13.877 & 14.879 & 16.469 & 17.522 & 17.416 \\
0.25 & 13.599 & 13.853 & 14.857 & 16.468 & 17.495 & 17.315 \\
0.30 & 13.595 & 13.831 & 14.836 & 16.467 & 17.468 & 17.214 \\
0.35 & 13.590 & 13.810 & 14.816 & 16.465 & 17.438 & 17.116 \\
0.40 & 13.583 & 13.792 & 14.797 & 16.463 & 17.410 & 17.019 \\
\hline
\end{tabular}


Table 4. Variation of cutoff frequency for different modes for the outer deformation of both vertical sides.

\begin{tabular}{lllllll}
\hline Shift & \multicolumn{7}{c}{ Frequency $(\mathrm{GHz})$} \\
\cline { 2 - 7 }$L(\mathrm{~cm})$ & $\mathrm{TM}_{011}$ & $\mathrm{TM}_{110}$ & $\mathrm{TM}_{111}$ & $\mathrm{TM}_{012}$ & $\mathrm{TM}_{112}$ & $\mathrm{TM}_{210}$ \\
\hline 0.05 & 13.607 & 13.928 & 14.926 & 16.469 & 17.574 & 17.620 \\
0.10 & 13.607 & 13.872 & 14.882 & 16.468 & 17.533 & 17.416 \\
0.15 & 13.607 & 13.817 & 14.843 & 16.465 & 17.494 & 17.211 \\
0.20 & 13.607 & 13.765 & 14.808 & 16.461 & 17.457 & 17.014 \\
0.25 & 13.607 & 13.714 & 14.777 & 16.453 & 17.422 & 16.826 \\
0.30 & 13.606 & 13.666 & 14.749 & 16.437 & 17.388 & 16.656 \\
0.35 & 13.606 & 13.621 & 14.723 & 16.388 & 17.355 & 16.528 \\
0.40 & 13.606 & 13.577 & 14.700 & 16.269 & 17.323 & 16.479 \\
\hline
\end{tabular}

where

$$
S_{i j}=\int\left[\frac{\partial F_{i}}{\partial x} \frac{\partial F_{j}}{\partial x}+\frac{\partial F_{i}}{\partial y} \frac{\partial F_{j}}{\partial y}+\frac{\partial F_{i}}{\partial z} \frac{\partial F_{j}}{\partial z}\right] \mathrm{d} x \mathrm{~d} y \mathrm{~d} z
$$

and

$$
T_{i j}=\int F_{i} F_{j} \mathrm{~d} x \mathrm{~d} y \mathrm{~d} z .
$$

Here $F_{i}$ is the mapping function due to the $i$ th node and integrations are over the mesh element surface.

The functional for the whole region $\Omega$ is given by

$$
\Pi=\frac{1}{2}\left\{E_{z}\right\}^{T}[S]\left\{E_{z}\right\}-\frac{1}{2} K^{2}\left\{E_{z}\right\}^{T}[T]\left\{E_{z}\right\} .
$$

The condition that variation of $\Pi$ must be minimum i.e. zero, gives

$$
[S]\left\{E_{z}\right\}-K^{2}[T]\left\{E_{z}\right\}=0 \text {. }
$$

Equation (17) is the matrix equation to be solved to get eigenvalues and eigenvectors.

\section{Numerical calculations for microstrip resonator}

A microstrip resonator of sides $2.4 \mathrm{~cm} \times 1.2 \mathrm{~cm} \times 2.8 \mathrm{~cm}$ is considered. The deformation for each case is shown in figure $1(b-f)$.

Table 5. Variation of cutoff frequency for different modes for the inner deformation of one vertical side and outer deformation of other vertical side.

\begin{tabular}{lllllll}
\hline Shift & \multicolumn{7}{c}{ Frequency $(\mathrm{GHz})$} \\
\cline { 2 - 7 }$L(\mathrm{~cm})$ & $\mathrm{TM}_{011}$ & $\mathrm{TM}_{110}$ & $\mathrm{TM}_{111}$ & $\mathrm{TM}_{012}$ & $\mathrm{TM}_{112}$ & $\mathrm{TM}_{210}$ \\
\hline 0.05 & 13.606 & 13.987 & 14.976 & 16.470 & 17.615 & 17.816 \\
0.10 & 13.603 & 13.991 & 14.97 .3 & 16.471 & 17.605 & 17.820 \\
0.15 & 13.599 & 13.996 & 14.969 & 16.472 & 17.588 & 17.827 \\
0.20 & 13.593 & 14.004 & 14.964 & 16.474 & 17.565 & 17.836 \\
0.25 & 13.586 & 14.013 & 14.956 & 16.476 & 17.537 & 17.848 \\
0.30 & 13.577 & 14.025 & 14.948 & 16.479 & 17.505 & 17.862 \\
0.35 & 13.568 & 14.037 & 14.938 & 16.482 & 17.469 & 17.879 \\
0.40 & 13.557 & 14.051 & 14.926 & 16.485 & 17.430 & 17.898 \\
\hline
\end{tabular}



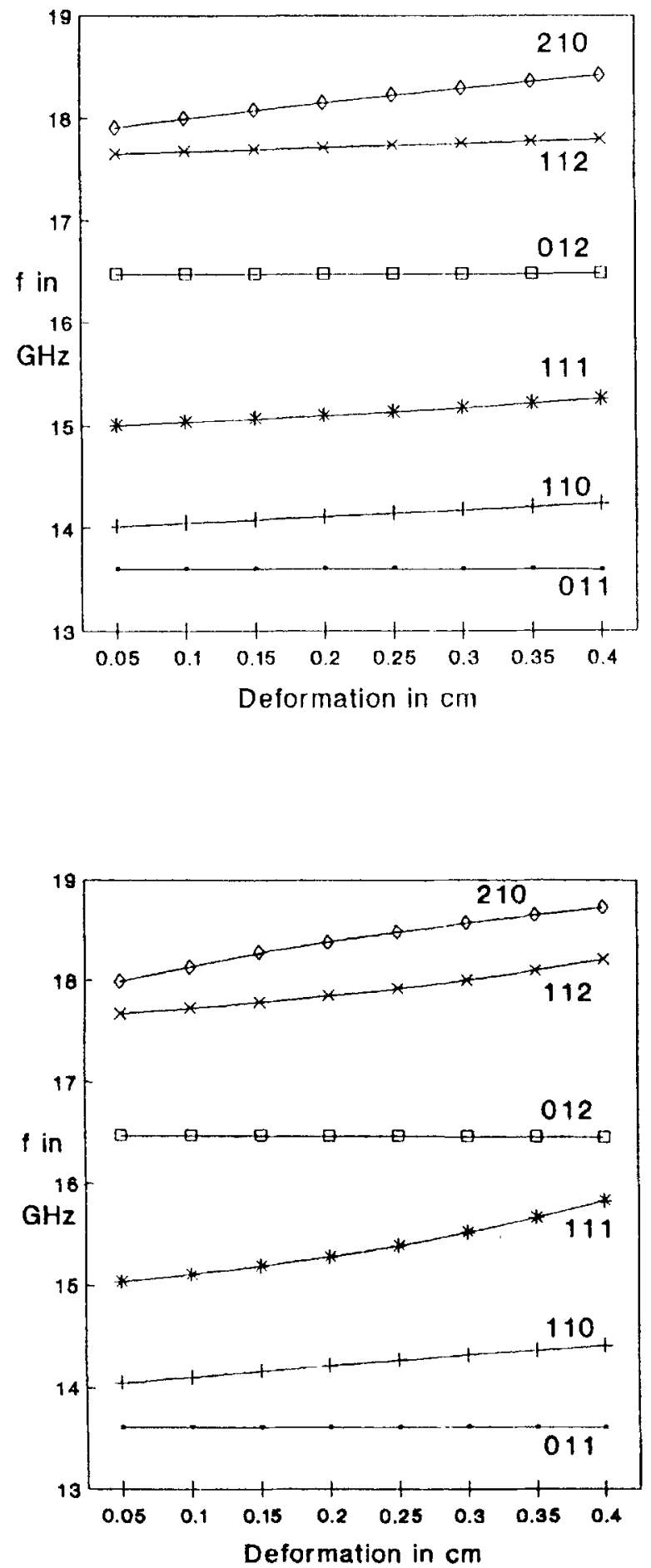

Figure 2. Variation of cutoff frequencies for different modes for the inner deformation of one vertical side of back surface.
Figure 3. Variation of cutoff frequencies for different modes for the inner deformation of both vertical sides of back surface. 

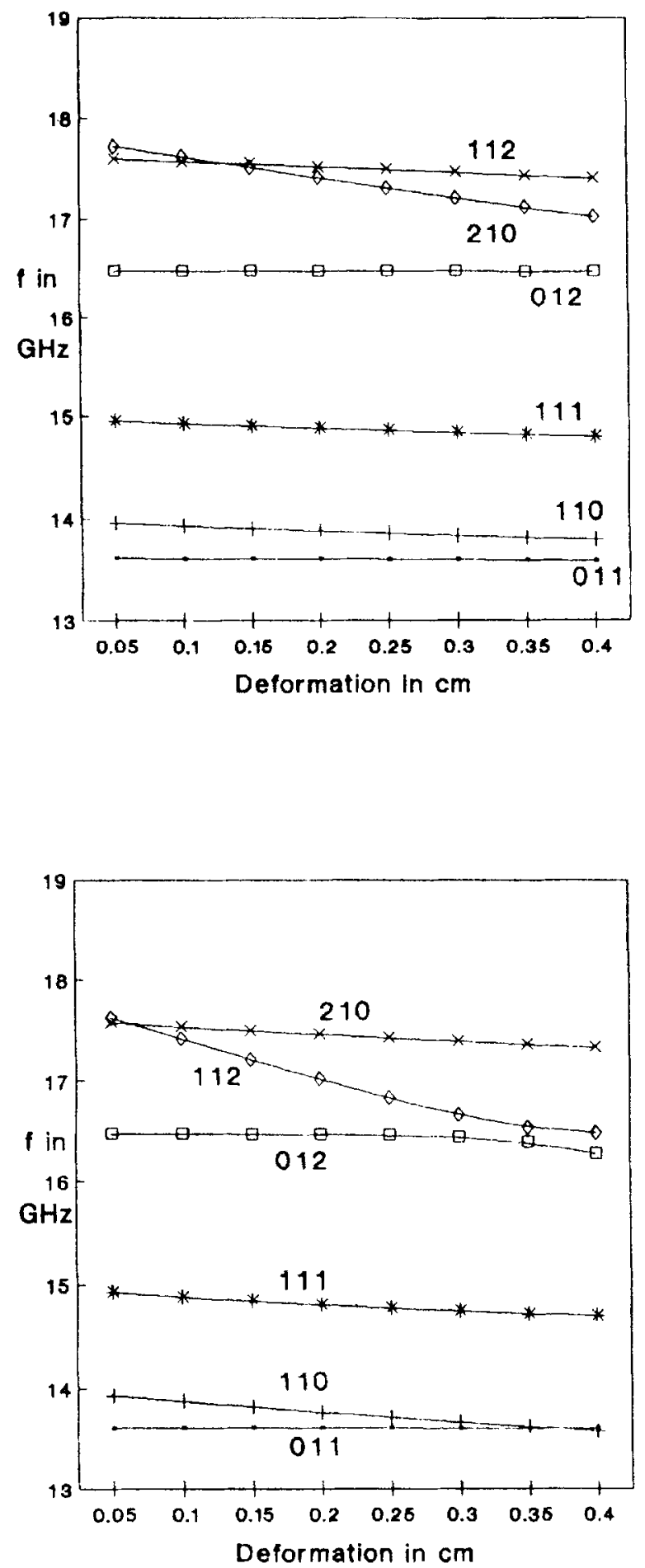

Figure 4. Variation of cutoff frequencies for different modes for the outer deformation of one vertical side of back surface.

Figure 5. Variation of cutoff frequencies for different modes for the outer deformation of both vertical sides of back surface. 


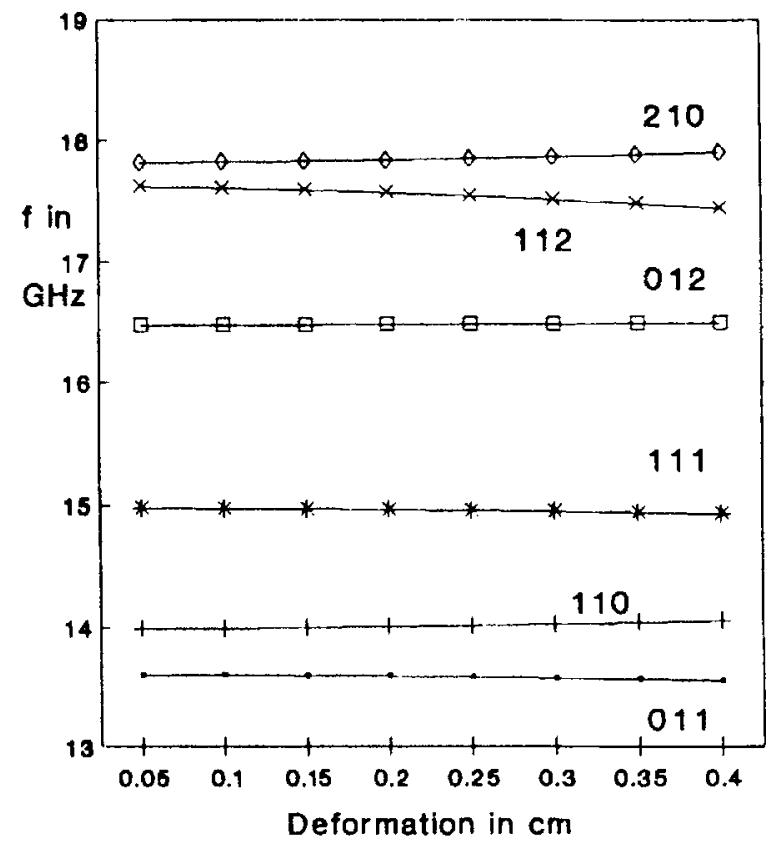

Figure 6. Variation of cutoff frequencies for different modes for the inner deformation of one vertical side and outer deformation of other vertical side of back surface.

(i) First case: Left vertical side of the back surface of the microstrip resonator is shifted inward by $0.05 \mathrm{~cm}$.

(ii) Second case: Both vertical sides of the back surface of the microstrip resonator are shifted inward by $0.05 \mathrm{~cm}$.

(iii) Third case: Right vertical side of the back surface of the microstrip resonator is shifted outward by $0.05 \mathrm{~cm}$.

(iv) Fourth case: Both vertical sides of the back surface of the microstrip resonator are shifted outward by $0.05 \mathrm{~cm}$.

(v) Fifth case: Left vertical side of the back surface is shifted inward by $0.05 \mathrm{~cm}$ and the right vertical side is shifted outward by $0.05 \mathrm{~cm}$.

In each case, for every shift, eigenvalues and eigenvectors are obtained. $\mathrm{TM}_{011}, \mathrm{TM}_{110}$, $\mathrm{TM}_{111}, \mathrm{TM}_{012}, \mathrm{TM}_{112}$ and $\mathrm{TM}_{210}$ modes are identified using field plots. These eigenvalues are the values of $\omega^{2} \mu_{0} \varepsilon_{0}$. From this, the corresponding cutoff frequencies are calculated in gigahertz.

For every shift, in each case, variation of cutoff frequency with the shift for the above modes is given in tables 1-5 and shown graphically in figures 2-6.

\section{Conclusions}

For the inner deformation of one or both vertical sides of the back surface, the cutoff frequencies increase with the shift for all the modes, except for $\mathrm{TM}_{011}$ and $\mathrm{TM}_{012}$ where they are almost constant. 
For the outer deformation of one or both vertical sides of the back surface, the cutoff frequencies decrease with the shift for all the modes.

For the inward deformation of one vertical side and outward deformation of the other vertical side, values of cutoff frequency for the $\mathrm{TM}_{011}, \mathrm{TM}_{111}$ and $\mathrm{TM}_{112}$ modes decrease with the shift, while for the $\mathrm{TM}_{110}, \mathrm{TM}_{012}$ and $\mathrm{TM}_{210}$ modes they increase.

\section{References}

Akin J E 1988 Application and implementation of finite element method (New York: Academic Press)

Helszajn J, James D S, Nisbet W T 1979 Circulators using planar triangular resonators. IEEE Trans. Microwave Theory Tech. 27: 188-193

Kalmase G M, Patil P B 1994a Finite element analysis of triangular patch microstrip resonator with shape deformation. Indian J. Phys. B68: 341-345

Kalmase G M, Patil P B 1994b Finite element analysis of rectangular patch microstrip resonator with shear deformation. Indian J. Pure Appl. Phys. 32: 899-901

Lyon R W, Helszajn J 1982 A finite element analysis of plane circulators using arbitrary shaped resonators. IEEE Trans. Microwave Theory Tech. 30: 1964-1974

Pintzos S G, Pregla R 1978 A simple method for computing the resonant frequencies of microstrip ring resonator. IEEE Trans. Microwave Theory Tech. 26: 809-813

Reddy J N 1986 An introduction to finite element method (New York: McGraw Hill)

Wolff I, Knoppik N 1974 Rectangular and circular microstrip capacitors and resonator. IEEE Trans. Microwave Theory Tech. 22: 857-864

Wolff I, Menzel W 1975 The microstrip ring resonator. IEEE Trans. Microwave Theory Tech. 23: $441-444$ 